\title{
A Massively Parallel Reservoir Simulator
}

\author{
Johnny Frøyen and Magne Espedal \\ Department of Mathematics, University of Bergen
}

Paper presented at the $5^{\text {th }}$ European Conference on the Mathematics of Oil Recovery, Leoben, Austria, 3-6 Sept.1996

\begin{abstract}
A two-phase sequential IMPLICIT three dimensional reservoir simulator has been developed for massively parallel computer systems. The parallel algorithm is based on recently developed domain decomposition methods. The simulator has been tested using a 16384 processor MasPar MP-4.10.

The simulator building blocks are robust Krylov space iterations using Additive Schwarz as the preconditioner. This approach naturally supports a distributed data structure with overlapping subdomains. In the simulator each subdomain is treated as a small reservoir in its own right, and each subdomain communicates with the neighboring subdomains through boundary conditions. Previous work has shown that the use of minimal overlap is sufficient and computationally nearly optimal for these problems.
\end{abstract}

\section{INTRODUCTION}

This paper describes and reports results obtained with a prototype of a new massively parallel black oil simulator. An important part for the project has been to implement a numerical simulator on a Massively. Parallel Computer. Such computers are able to handle huge amount of data, thus giving us the opportunity of simulating big heterogeneous reservoir problems.

In commercial numerical reservoir simulators the finite difference method is the most commonly used discretization technique, and the upstream weightening method is used to stabilize the convective flow problem. These methods introduce artificial numerical dispersion which are at the order of the grid size. On field-scale this limits the accuracy of the predicted flow pattern and the predicted hydrocarbon recovery. Techniques which control the numerical dispersion have had limited succsess in ordinary reservoir studies done by oil companies. The computing power of parallel computers now allows us to test and implement new numerical solution methods that greatly reduce numerical diffusion. ${ }^{23}$

The basic idea behind the parallelization strategy is to exploit data locally by using a data structure where local in the physical model implies local in the computer representation. The domain decomposition approaches that we have followed, effectively separates short and long range communication into two distinct phases in each precondition step. It is then easier to analyze the performance of the method and to adjust the notion of locality with respect to

\footnotetext{
${ }^{0}$ References and illustrations at end of paper.
} 
the target computer at hand. Our goal is an algorithm that is portable and efficient in a distributed memory system. A simulator based on the algorithms and ideas described in this paper should adapt well to hierarchal memory, cache based single processor systems, as well as distributed memory parallel computers with a possible wide range of performance characteristics.

\section{THE SOLUTION STRATEGY}

Neglecting compressibility, two-phase immiscible flow in a porous medium can be described by the following set of partial differential equations ${ }^{1}$

$$
\begin{aligned}
& \nabla \cdot \mathbf{u}=q(\mathbf{x}, t) \\
& \mathbf{u}=-\mathbf{A}(S, \mathbf{x}) \cdot(\nabla p+g \rho \mathbf{k}), \\
& \phi \frac{\partial}{\partial t} S+\nabla \cdot\left[f(S) \mathbf{u}+f_{g}(S) \mathbf{u}_{g}\right] \\
& \quad-\epsilon \nabla \cdot(\mathbf{D}(S, \mathbf{x}) \cdot \nabla S)=q_{w}(\mathbf{x}, t) .
\end{aligned}
$$

Here $\mathbf{u}$ is the total Darcy velocity, $p$ the total fluid pressure, $S$ denotes the saturation of water, $g$ is the gravity acceleration and $\epsilon$ is a parameter scaling the diffusion. We assume that the absolute permeability tensor is diagonal

$$
\mathbf{K}(\mathbf{x}) \doteq k_{x}(\mathbf{x}) \mathbf{i i}+k_{y}(\mathbf{x}) \mathbf{j} \mathbf{j}+k_{z}(\mathbf{x}) \mathbf{k} \mathbf{k} .
$$

Let $\lambda_{i}, i=w$, o denote the mobility of water and oil respectively. We then have:

$$
\begin{aligned}
& \mathbf{A}(S, \mathbf{x})=\mathbf{K}(\mathbf{x})\left(\lambda_{w}+\lambda_{o}\right), \\
& \mathbf{D}(S, \mathbf{x})=\mathbf{K}(\mathbf{x}) \frac{\lambda_{w} \lambda_{o}}{\lambda_{w}+\lambda_{o}} \frac{d p_{c}}{d S} \\
& f(S)=\frac{\lambda_{w}}{\lambda_{w}+\lambda_{o}}, \\
& f_{g}(S)=\lambda_{o} f(S) \\
& \rho=\frac{\lambda_{w} \rho_{w}+\lambda_{o} \rho_{o}}{\lambda_{w}+\lambda_{o}} \\
& \mathbf{u}_{g}=-\left(\rho_{w}-\rho_{o}\right) \mathbf{k}
\end{aligned}
$$

where $p_{c}$ is the capillary pressure and $\rho_{i}, i=w, o$ denotes the density of water and oil. The mobilities and the capillary pressure are assumed to be known functions of the water saturation.

To solve this system, we have used sequential time stepping. Substituting (2) into (1) we get the second order elliptic equation

$$
-\nabla \cdot A(S, \mathbf{x})(\nabla p+g \rho \mathbf{k})=q(\mathbf{x}, t)
$$

In our solution procedure we first solves for the pressure (Eq. (11)) with saturation kept fixed. The Darcy-velocity is then found using eq. (2). After the velocity has been advanced in time, the saturation is updated.

Eq (11) is discretized using a standard Galerkin finite element formulation.

A straight forward differentiation of the pressure would result in a velocity field with error of linear order (one order lower than the pressure). We have computed the velocity field based on flux consideration. For sufficient regular grid such methods are offering superconvergence. ${ }^{27}$

The solution of (3) is based on the operator splitting introduced by Espedal and Ewing. ${ }^{18}$ First the fractional function $\mathbf{F}(S, \mathbf{x})=f(S) \mathbf{u}+f_{g}(S) \mathbf{u}_{g}$ is divided into two parts

$$
\begin{aligned}
F(S, \mathbf{x}) & =\bar{f}(S, \mathbf{x}) \mathbf{u}+b(S, \mathbf{x}) \mathbf{u} S+f_{g}(S) \mathbf{u}_{g} \\
& =\bar{f}(S, \mathbf{x}) \mathbf{u}+\mathbf{b}(S, \mathbf{x}) S
\end{aligned}
$$

such that $\bar{f} \mathbf{u}$ gives the unique physical velocity for an established shock, and the nonlinear part $\mathbf{b}$ balances the diffusion at the shock. Then operator-splitting is used to treat convection and diffusion separately. For each time-step, two equations are solved

$$
\begin{aligned}
& \phi \frac{\partial \bar{S}}{\partial t}+\nabla \cdot(\bar{f}(\bar{S}) \mathbf{u})=0 \\
& \phi \frac{\partial S}{\partial \tau}+\left(\bar{f}^{\prime}(S)-\bar{f}^{\prime}(\bar{S})\right) \mathbf{u} \cdot \nabla S \\
& \quad+\nabla \cdot[\mathbf{b}(S, \mathbf{x}) S-\epsilon \mathbf{D}(S, \mathbf{x}) \cdot \nabla S]=0 .
\end{aligned}
$$

Where

$$
\frac{\partial}{\partial \tau}=\frac{\partial}{\partial \tau(\bar{S})}=\frac{\partial}{\partial t}+\frac{1}{\phi} \bar{f}^{\prime}(\bar{S}) \mathbf{u} \cdot \nabla .
$$

The characteristic system associated with equation (12) is given by

$$
\begin{aligned}
\phi \frac{d \bar{S}}{d \tau} & =\phi \frac{\partial \bar{S}}{\partial t}+\mathbf{u} \cdot \nabla \vec{f}(\bar{S})=0 \\
\frac{d t}{d \tau} & =\phi \\
\frac{d \mathbf{x}}{d \tau} & =\bar{f}^{\prime}(s) \mathbf{u}
\end{aligned}
$$

A solution to this problem is found by intergating backward along the characteristics. This gives us a first approximation $\bar{S}$ to $S$. By discretizing Eq. (13) 
in time and linerizing it by expanding with respect to $\bar{S}$, we get.

$$
\begin{aligned}
& S+\frac{\Delta t}{\phi} \nabla \cdot(\mathbf{b}(\bar{S}, \mathbf{x}) S) \\
& -\frac{\Delta t \epsilon}{\phi} \nabla \cdot(\mathbf{D}(\bar{S}, \mathbf{x}) \cdot \nabla S)=\bar{S}
\end{aligned}
$$

More details on this solution procedure is carefully worked out in. ${ }^{10,23,26}$

To discretize this equation equation we have used a Petrov-Galerkin formulation.

In the above solution procedure it is assumed that $\bar{S}$ is a fairly good approximation to $S$. How good this approximation is, depends on several factors, such as the time discretization parameter and influence of gravity. The error introduced in (15) due to differences between $\bar{S}$ and $S$ can be compensated for by iterate on (13). As iteration procedure, we have used a Picard iteration. Let $S_{0}=\bar{S}$. The iteration procedure procedure can be written

$$
\begin{aligned}
& \text { for } k=0,1,2, \ldots m \\
& S_{k+1} \\
& +\frac{\Delta t}{\phi} \nabla \cdot\left\{\mathbf{b}\left(S_{k}, \mathbf{x}\right) S_{k+1}-\epsilon \mathbf{D}\left(S_{k}, \mathbf{x}\right) \nabla S_{k+1}\right\} \\
& =\bar{S}-\frac{\Delta t}{\phi}\left\{\bar{f}^{\prime}\left(S_{k}\right)-\bar{f}^{\prime}(\bar{S})\right\} \mathbf{u} \cdot \nabla S_{k}
\end{aligned}
$$

\section{THE MASPAR COMPUTER SYS-}

\section{TEM}

MasPar is a massively parallel Single Instruction, Multiple Data computer system (SIMD). It consists of four subsystem:

- a front-end graphics workstation that runs the UNIX operating system

- a high-speed I/O subsystem

- the PE array

- the ACU

The last three components are all included in the data parallel unit (DPU). The PE array consist of a numbers processor elements arranged in a 2-dimensional matrix. The computer we have used consist of 128 $\times 128$ processor elements (PEs). Each PE is a $32-$ bit load/store arithmetic processor with dedicated resister space and a RAM of $64 \mathrm{~K}$. Instructions are sent from the array control unit (ACU) to every PEs simultaneously. Exchanging of data between neighbouring processors can be done by fast Xnet communication routines. Between two arbitrary PEs more expensive Global Router communication is used.

\section{DATA STRUCTURE.}

Domain decomposition is an important part of the solution procedure for the linear systems that results from the the finite element discretization of (11) and (15). It is therefore natural to consider a data structure where the reservoir (domain) is partitioned into many subdomains (subreservairs), and each subdomain is mapped onto one PE in the PE array. Each processor can then be considered as a reservoir simulator of its own carrying the entire data structure of a subdomain. The boundary data is duplicated in subdomains that are neighbours. The stiffness matrices for (11) and (15) are assembled and stored as part of the local data structure for each subdomain.

Most of the communication needed in the linear solvers will be between neighbouring subdomains. The communication costs would therefore be minimal if neighbouring subdomains were mapped onto neighbouring processor. This is, however, not possible due to the 2-dimensional architecture of MasPar. Instead we have divide the PE array into four subarrays of equal size. Each subarray represents a horizontal layer. By doing the mapping this way we can still use fast communication between neighbouring subdomains in the same horizontal layer. Between neighbouring subdomains in different layers however, more expensive communication is required.

\section{ALGORITHMS AND IMPLEMENTA- TION.}

Discretizing (11) and (15) results in two large sparse linear systems. These systems are solved by Krylov space iterations together with an Additive Schwarz preconditioner (ASM). ASM are domain decomposition method using overlapping subdomains. There is an extensive literature on Additive Schwarz preconditioners, see $e^{3,7-9,13-15}$ and references therein. The ASM preconditioners can be viewed as a generalization of block Jacobi iterations, ${ }^{17}$ and it can be derived in the following way. The reservoir $\Omega$ is divided into $N$ overlapping subdomains $\Omega_{i}$. Let $R_{i}$ be the restriction matrix that project a grid function $u$ onto the subdomain $\Omega_{i}$

$$
u_{i}=R_{i} u
$$

Also let $A x=b$ be the linear problem defined on the global domain $\Omega$. The first ASM preconditioner step consists of solving

$$
A_{i} x_{i}=b_{i}
$$


where

$$
A_{i}=R_{i} A R_{i}^{T}, \quad x_{i}=R_{i} x, \quad b_{i}=R_{i} b
$$

This gives us a first approximation

$$
\tilde{x}=\sum_{i=1}^{N} R_{i}^{T} x_{i}
$$

to $x$. This part smooths out the high frequency components of the error, but do a poor job of correcting the low frequency components. Such effects are of global nature. A residual of low frequency in one part of the reservoir will affect the error everywhere. To resolve the low frequency components of the error we must have some mechanism for the global communication of information at each iteration. Therefore we introduce the stiffness matrix $A_{c}$ coming from discretizing on the global domain, with element size equal to the subdomains. Now let $R_{0}$ be the restriction such that

$$
u_{c}=R_{0} u
$$

and $u_{c}$ is a grid function defined on the coarse subdomain grid. Our next ASM preconditioner step then consist of solving

$$
A_{c} x_{c}=b_{c}, \quad b_{c}=R_{0} b
$$

finally we update the approximate solution

$$
\tilde{x}=\tilde{x}+R_{0}^{T} x_{c}
$$

Defining $B_{i}=R_{i}^{T} A_{i}^{-1} R_{i}, i=1, \ldots N B_{0}=R_{0}^{T} A_{c}^{-1} R_{0}$ the ASM preconditioner can be written:

$$
B=\sum_{i=0}^{N} B_{i}
$$

This class of domain decomposition preconditioner can be shown to have optimal convergence properties. This means that if the overlap between subdomains is fixed, then the number of iterations are bounded, independent of the discretization parameters $h$ (the fine grid), $H$ (the coarse grid) and the ratio $H / h$. More information on the theoretical properties of the method can be found in the references listed above. In our implementation, we violate the assumption that the overlap should be independent of $h$, neighbouring subdomains have only one line and grid element in common. This leads more iterations, but extensive experiments ${ }^{20,5,28}$ and theoretical results ${ }^{16}$ indicate that such an approach may still be an advantage when the amount of data stored and the duplicated computation in the overlap area is taken into account. That is, small overlap tends to minimize the overall computational time.
One attractive feature of this method is that inexact solvers may be used to solve the local subproblems instead of direct solvers. Therefore it is possible to use inexact solvers which fits the actual problem.

\section{The pressure and the velocity calcula- tions}

This part of the simulator is quite naturally divided into three steps.

- The set up of the stiffness matrix and the right hand side of the equation.

- The solution of the linear system

- The calculation of the velocity field

As already mentioned equation (11) is discretized by a standard Galerkin formulation. The geometric objects are three dimensional boxes. The finite dimensional three-linear space of functions is choosen to be the set of three-linear functions on each geometric object.

All the permeabilities are stored locally and the calculation of $\mathbf{A}(s, \mathbf{x})$ in (11) is a completely local calculation. Therefore the set up of the small stiffness matrices $A_{i}$ and the coarse matrix $A_{c}$ can be carried out within each local subdomain.

The resulting linear system is symmetric, positive definite and the appropriate Krylov space method is Conjugate Gradients. ${ }^{19}$ All Krylov space methods together with a preconditioning step, have three main computational kernels.

- The preconditioning step

- The matrix vector calculation

- The inner product calculation

The local subproblems $B_{i}, i=1, \ldots, N$ are solved approximately using a fixed but small number of SSOR iterations. $^{19}$ The application of $B^{\prime}=\sum_{i=1}^{N} B_{i}$ to a vector requires communication between nearest neighbour data structures when the local boundary values are updated. The coarse grid matrix $B_{0}$ requires exchange of data between all subdomains. The coarse problem is solved by a multigrid solver. In the multigrid solver we use a small but fixed number of $\mathrm{V}$ cycles. ${ }^{6,21}$ This gives structured long range communication pattern. Previous work on this type of ASM preconditioner shows that it is very robust with respect to large variation in the permeability. 4,28

The matrix vector product is calculated locally within each subdomain and this part of the solver is fast. In the inner product step most of the calculation can be done within each subdomain, but at the end 
all the computed numbers have to be merged into one single number. To do this all processors have to communicate and this is the only sequential step in this part of the code.

Finally the total velocity $\mathbf{u}$ is determined based on flux consideration. Again this it is a local calculation. The time spent in this part of the code is negligible.

\section{The hyperbolic solver}

The numerical solution of (14) is done by the Modified Method of Characteristics (MMOC). ${ }^{12}$ We find the saturation $S(\mathrm{x})$ at time level $t^{n+1}$ by tracking backward along a characteristic finding the saturation $S(\overline{\mathbf{x}})$ at point $\overrightarrow{\mathbf{x}}$ at time level $t^{n}$. This may be written:

$$
\overline{\mathbf{x}}=\mathbf{x}-\int_{\dot{\tau}^{n}}^{\tau^{n+1}} \mathbf{v} \bar{f}^{\prime}(S, \mathbf{x}) d \tau
$$

Since we have integrated backward along a characteristic we know that the saturation at a point $\mathbf{x}$ at time level $t^{n+1}$ has to be equal the saturation at point $\overline{\mathbf{x}}$ at time level $t^{n}$. Starting by a value $S_{0}$ and the corresponding fractional flow value $\bar{f}^{\prime}\left(S_{0}\right)$ we trace back and get a point $\overline{\mathbf{x}}$, if the saturation $s(\overline{\mathbf{x}})$ is equal $S_{0}$ then we stop, otherwise we do bisection on $\bar{f}^{\prime}\left(S_{0}\right)$ and find the corresponding saturation value and continue. The process starts by using the saturation at the old time level.

The integration backward is performed by dividing the integration interval into a number of subinterval, within each subinterval the velocity is supposed to be constant.

This algorithm is carried out for all grid points within each local subdomain, but the calculation depends on the subdomain size and the length of the time step. Access data along characteristic may force us to use general type of communication between processors, and this type of communication is often very slow.

The fractional function $f$ used in our experiments will typically have an $S$ shaped form. The $\bar{f}$ function will then be defined according to the following rules:

$$
\begin{aligned}
& \bar{f}=\text { the convex hull of } f \text { if } \nabla \bar{S} \cdot \mathbf{u}<0 \\
& \bar{f}=\text { the concave hull of } f \text { if } \nabla \bar{S} \cdot \mathbf{u}>0
\end{aligned}
$$

To define the correct $\bar{f}$ function we first assume that the saturation $S^{n}$ at the old time level $t^{n}$ is "monotone along the characteristics. We then choose $\bar{f}$ to be the convex hull of $f$. Stating at a point $\mathbf{x}$ we integrate backward along the characteristic, to obtain a solution $\tilde{S}(\mathbf{x})$ to $(12)$. If $\tilde{S}-S^{n}>0, \tilde{S}$ will, due to the monotony assumption, be the correct solution to
(12). If not we choose $\bar{f}$ to be the concave hull of $f$. We then repeat the integration process to obtain the correct solution to (12).

\section{The elliptic saturation solver}

The last computational step is to solve equation (15). This equation is discretized with finite element method, using rectangular boxes. A Petrov-Galerkin formulation is used to better represent the convective term. More detail on this can be found in. $2,11,22,24$ The final linear equation is non-symmetric and the Krylov space method BiCGstab ${ }^{29}$ was found to perform satisfactory.

Several computational steps are similar to the pressure solver, and again we use an Additive Schwarz preconditioner. As subdomain solver we have used the Krylov space method GMRES. ${ }^{25}$ The main difference between the preconditioner for the pressure equation and the preconditioner for the saturation equation is that in the latter we have no coarse grid solver. In the saturation equation the previous hyperbolic step should (if our splitting is successful ) essentially move the oil-water front to its new position. Equation (15) serves as a correction in order to fully represent the fractional flow function and to account for the diffusion term.

\section{RESULTS AND DISCUSSION}

In this section we will report some of the numerical experiments we have done with the new prototype simulator. The reservoir will be a rectangular box with one injector and one producer. We have tested the simulator for both homogeneous and heterogeneous reservoir with no-flow boundary condition. In all of our experiments we stop the the Krylov space iterations when the discrete $L_{2}$ norm of the residual is reduced by a factor of $10^{-6}$. The simulations are stopped when 20 percent of the oil original in place are produced $(0.20$ $\mathrm{PV}$ ). In the simulations the number of processors and the number of subdomains are equal, all subdomains have the same number of nodes and we have used $128 \times 128 \times 64$ grid blocs.

In table 1 see how much of the total computing time different parts of the simulator uses.

When integrating backward along the characteristic, communication between arbitrary subdomains (processors) are needed. Such communication is expensive. Most of the computing time in the Hyperbolic solver comes from the communication.

As mentioned above we have not used a multigrid in the preconditioner for the saturation equa- 
tion. However the problem is dependent on a relatively good subdomain solver. When we used SSOR the problem did not converge. GMRES converged, but this methood needs to store all the search directions from the previous iterations. Due to the small RAM of the processors, we have only used two iteration in each preconditioner step. Better ways of constructing subdomains solvers may improve this part of the simulator.

Below we have plotted the saturation profiles for different simulations. The profiles are given along the diagonal of the horizontal plane of the three dimensional simulations. In figures (1)-(3) we have plotted the profile for three different gravity scaling. In the first case gravity forces are excluded. In the third case gravity is the dominated force. In the next tests we have run simulations on a heterogeneous reservoir. The $K x$ and $K y$ are constant, while $K z$ varies as shown in figure 4. Gravity forces are acting along the Z-Axis.

\section{CONCLUSION.}

A reservoir simulator based on operator splitting and domain decomposition has been implemented on a MasPar MP-4.10 computer system. The reservoir was divided into many subdomains and each subdomain has a data structure of an ordinary reservoir simulator. These small subdomains communicate with the rest of the reservoir through boundary conditions. This way of constructing the data structure is independent of the computer architecture. Numerical experiments shows that with this simulator we are able to run simulations with hundreds of thousand grid points.

\section{NOMENCLATURE.}

$\begin{array}{lll}\phi & : & \text { porosity } \\ S & : & \text { water saturation } \\ p & : & \text { global pressure } \\ \mathbf{u} & : & \text { global velocity } \\ q & : & \text { total production and injection rate } \\ q_{w} & : & \text { water injection rate } \\ \rho_{w} & : \text { density of water } \\ \rho_{0} & : \text { density of oil } \\ \lambda_{w} & : \text { mobility of water } \\ \lambda_{0} & : \text { mobility of oil } \\ g & : \text { gravity acceleration }\end{array}$

\section{References}

[1] K. Aziz and Antonin Settari. Petroleum reservoir simulation. Elsevier Applied Science Publishers, 1979 .

[2] J. W. Barrett and K. W. Morton. Approximate symmetrization and Petroy-Galerkin methods for diffusion-convection problems. Comp. Meth. in Appl. Mech. and Eng., 45, 1984.

[3] Petter E. Bjørstad. Multiplicative and Additive Schwarz Methods: Convergence in the 2 domain case. In Tony Chan, Roland Glowinski, Jacques Périaux, and Olof Widlund, editors, Domain Decomposition Methods, Philadelphia, 1989. SIAM.

[4] Petter E. Bjørstad and Morten Skogen. Domain decomposition algorithms of Schwarz type, designed for massively parallel computers. In Tony F. Chan, David E. Keyes, Gérard A. Meurant, Jeffrey S. Scroggs, and Robert G. Voigt, editors, Fifth International Symposium on Domain Decomposition Methods for Partial Differential Equations, Philadelphia, 1992. SIAM.

[5] Petter E. Bjørstad and Morten D. Skogen. Domain decomposition algorithms of schwarz type, designed for massively parallel computers. Technical Report 54, Dept. of informatics, University of Bergen, Høyteknologisenteret, N-5020 Bergen, Norway, 1991.

[6] William Briggs. A Multigrid Tutorial. SIAM, Philadelphia, 1987.

[7] Xiao-Chuan Cai. An additive Schwarz algorithm for nonselfadjoint elliptic equations. In Tony Chan, Roland Glowinski, Jacques Périaux, and Olof Widlund, editors, Third International Symposium on Domain Decomposition Methods for Partial Differential Equations, Philadelphia, 1990. SIAM.

[8] Xiao-Chuan Cai and Olof Widlund. Domain decomposition algorithms for indefinite elliptic problems. SIAM J. Sci. Statist. Comput., 13(1):243-258, January 1992.

[9] Xiao-Chuan Cai and Olof Widlund. Multiplicative Schwarz algorithms for some nonsymmetric and indefinite problems. SIAM J. Numer. Anal., 30(4):936-952, August 1993.

[10] H. K. Dahle. Adaptive Characteristic Operator Splitting Techniques for Convection-Dominated Diffusion in One and Two Space Dimension. 
PhD thesis, Dep. of Appl. Math., University of Bergen, Norway, 1988.

[11] L. Demkowicz and J. T. Oden. An adaptive characteristic Petrov-Galerkin finite element method for convection-dominated linear and non-linear parabolic problems in one space variable. Comp. Meth. in Appl. Mech. and Eng., 55, 1986.

[12] J. Douglas and T. F. Russel. Numerical methods for convection-dominated diffusion problems based on combining method of characteristic with finite elements or finite difference procedures. SIAM J, 19(5):871-885, 1982.

[13] Maksymilian Dryja. An additive Schwarz algorithm for two- and three-dimensional finite element elliptic problems. In Tony Chan, Roland Glowinski, Jacques Périaux, and Olof Widlund, editors, Domain Decomposition Methods, Philadelphia, 1989. SIAM.

[14] Maksymilian Dryja and Olof B. Widlund. An additive variant of the Schwarz alternating method for the case of many subregions. Technical Report 339, also Ultracomputer Note 131, Department of Computer Science, Courant Institute, 1987.

[15] Maksymilian Dryja and Olof B. Widlund. Additive Schwarz methods for elliptic finite element problems in three dimensions. In Tony F. Chan, David E. Keyes, Gérard A. Meurant, Jeffrey S. Scroggs, and Robert G. Voigt, editors, Fifth International Symposium on Domain Decomposition Methods for Partial Differential Equations, Philadelphia, 1992. SIAM.

[16] Maksymilian Dryja and Olof B. Widlund. Domain decomposition algorithms with small overlap. Technical Report 606, Department of Computer Science, Courant Institute, May 1992. To appear in SIAM J. Sci. Stat. Comput.

[17] Maksymilian Dryja and Olof B. Widlund. Some recent results on Schwarz type domain decomposition algorithms. Contemporary Mathematics, 157:53-61, 1994. In proceedings from the sixth international symposium on domain decomposition.

[18] M. S. Espedal and R. E. Ewing. Characteristic Petrov-Galerkin subdomain method for two- phase immiscible flow. Comp. Meth. in Appl. Mech. and Eng., 64, 1987.

[19] G. H. Golub and C. F. Van Loan. Matrix Computations. John Hopkins Univ. Press, 1989. Second Edition.

[20] W. Hackbusch, editor. . Parallel domain decomposition and iterative refinement algorithms. VIEWEG, 1990. Notes on Numerical Fluid Mechanics, Vol 31, Proceeding of the sixth GAMMA-Seminar, Kiel, January 19-21 1990.

[21] Wolfgang Hackbusch. Multigrid Methods and Applications. Springer, Berlin, 1985.

[22] C. Johnson. Numerical solution of partial differential equations by the finite element method. Cambridge University Press, 1987.

[23] Terje Kårstad. Massively parallel algorithms in reservoir simulation. PhD thesis, Department of Informatics, University of Bergen, September 1993.

[24] K. W. Morton. Finite Element Methods for None-Self-Adjoint Problems. Springer-Verlag, 1982. Lecture Notes in Mathematics 965.

[25] Y. Saad and H. Schultz. Gmres: A generalized minimal residual algorithm for solving nonsymmetric linear systems. SIAM, J, Sci. Stat. Comp., 7,1986 .

[26] O. Sævareid. On local Grid Refinement Techniques for Reservoir Flow Problems. PhD thesis, Dep. of Appl. Math., University of Bergen, Norway, 1990 .

[27] O. Sævareid. On consistent and accurate velocities from a picewise (bi-)linear pressure approximation. Technical report, Dep. of Applied Math. University of Bergen, To be published.

[28] M. D. Skogen. Parallel Schwarz methods. PhD thesis, University of Bergen, Dept. of Informatics, Høyteknologisenteret, N-5020 Bergen, Norway, 1992.

[29] H. A. van der Vorst. Bi-CGSTAB: A fast and smootly converging variant of $\mathrm{Bi}-\mathrm{CG}$ for solution of non-symmetric limear systems. SIAM J. Sci. Statist. Comput., 13, 1992. 


\begin{tabular}{|c|c|}
\hline Subroutine & \% computing time \\
\hline Presure & 20.3 \\
\hline Hyperbolic & 40 \\
\hline Diff & 39.7 \\
\hline
\end{tabular}

Table 1: Table shows how many \% of the total computing time the different parts of the program uses.

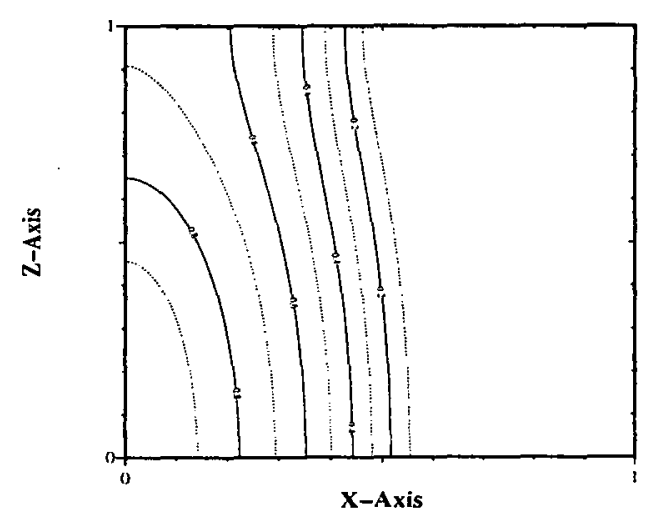

Figure 1: The saturation profile for a homogeneous reservoir. Gravity forces are excluded $\epsilon=0.05$.

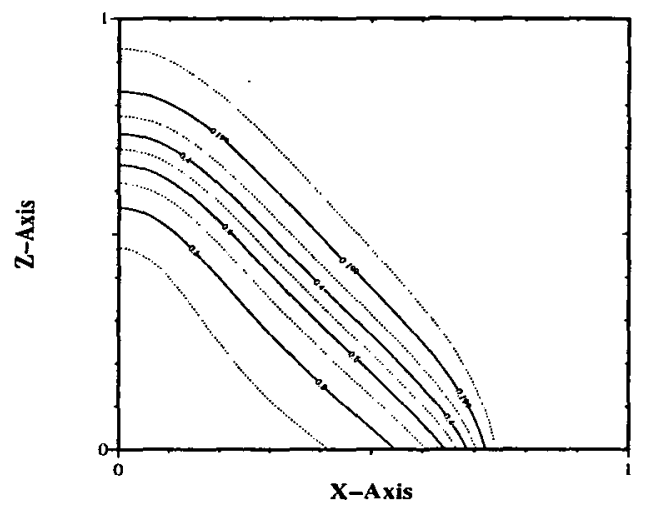

Figure 2: The saturation profile for a homogeneous reservoir. $\rho_{w}=0.5, \rho_{0}=0.4, \epsilon=0.05$

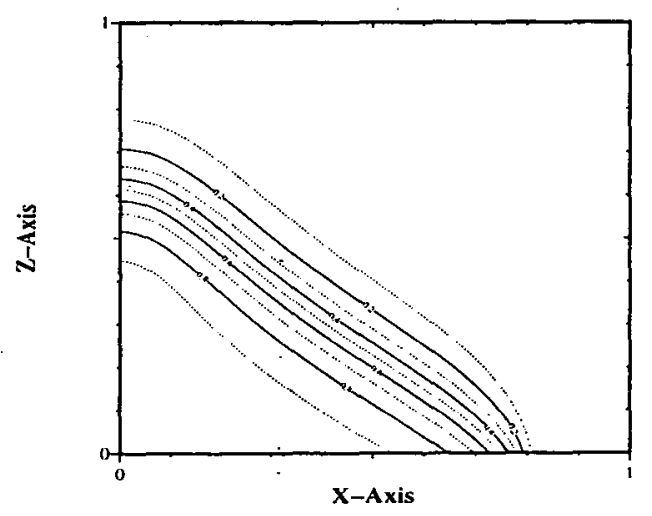

Figure 3: The saturation profile for a homogeneous reservoir. $\rho_{w}=1.5, \rho_{0}=1.2, \epsilon=0.05$ 


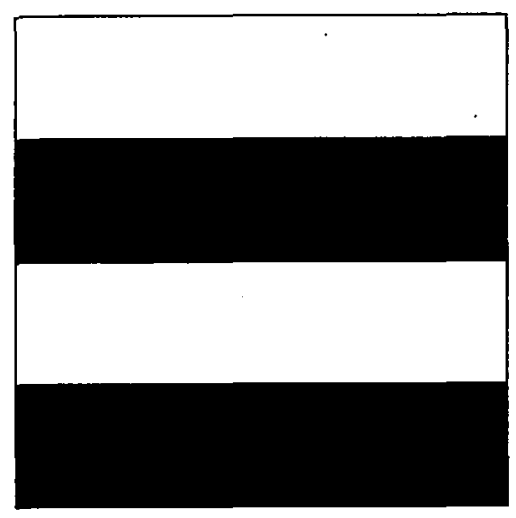

Figure 4: Permeability data for $K z . K z=1$ in dark area, $K z=0.1$ in bright area

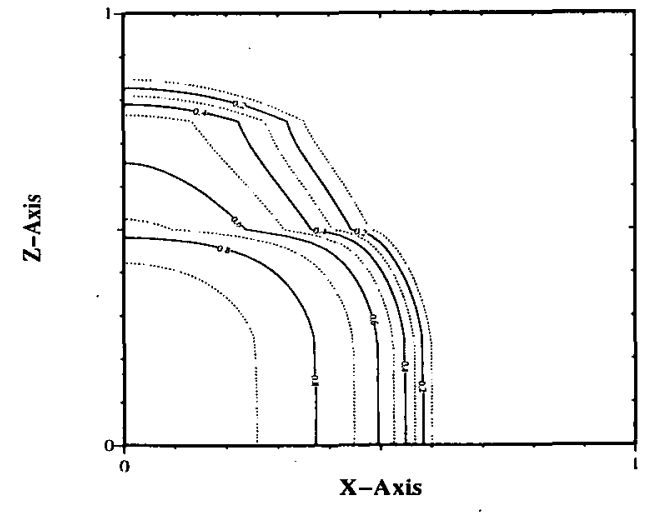

Figure 5: The saturation profile for a a heterogeneous reservoir. Gravity forces are excluded $\epsilon=0.02$

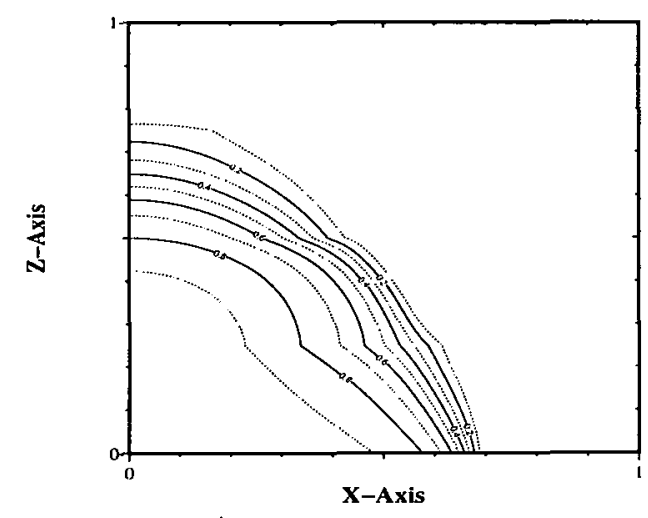

Figure 6: The saturation profile for a heterogeneous reservoir. $\rho_{w}=0.5, \rho_{0}=0.4, \epsilon=0.02$

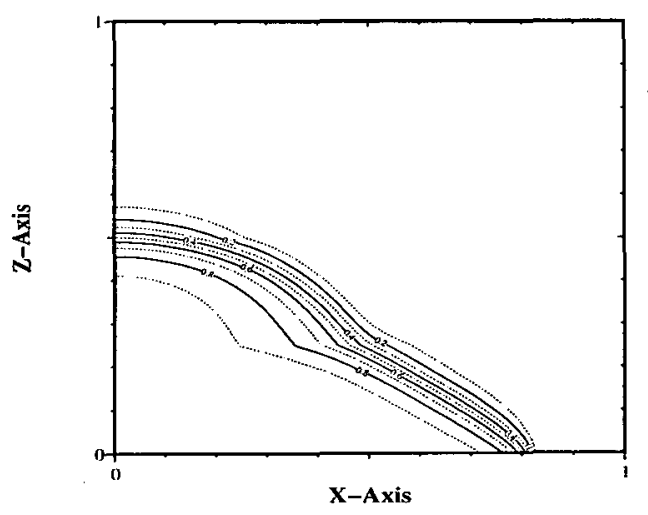

Figure 7: The saturation profile for a heterogeneous reservoir. $\rho_{w}=1.5, \rho_{0}=1.2, \epsilon=0.02$ 
